\title{
Análisis de la autogestión del aprendizaje del estudiante de grado: un caso de estudio en la modalidad a distancia
}

\author{
Analysis of self-management of undergraduate student learning: a case \\ study in the distance modality
}

Eulalia Beatriz Becerra García. ${ }^{1}$, Aracelly Fernanda Núñez Naranjo. ${ }^{2}$ \& Verónica Elizabeth Olalla Pardo. ${ }^{3}$

\begin{abstract}
. https://doi.org/10.33262/concienciadigital.v4i3.1.1811

Introduction. Due to the COVID-19 pandemic that is currently impacting humanity, people's lifestyles have changed drastically, one of the most affected sectors is education, for this reason the students programmed themselves to look for better learning strategies. Objective. The main objective of this work is to carry out an analysis of the selfmanagement techniques for undergraduate student, a case study in the on-line Methodology. A survey of 33 questions was applied to learn the self-management techniques for student learning, divided into four sections: planning, monitoring, control and motivation. Results. The main results are as follow: it was concluded that students of higher levels apply better strategies for self-management learning than students of middle and lower levels. Conclusions. The students of lower and middle levels do not have strategies that help to improve their self-learning, for which it is necessary another

1 Universidad Tecnológica Indoamérica, Carrera de Educación Básica, Ambato, Ecuador. eulaliabecerra@indoamerica.edu.ec, https://orcid.org/0000-0002-0677-7393..

2 Universidad Tecnológica Indoamérica, Carrera de Educación Básica, Ambato, Ecuador. fernandanunez@indoamerica.edu.ec, https://orcid.org/0000-0002-0185-5516.

3 Universidad Tecnológica Indoamérica, Carrera de Educación Básica, Ambato, Ecuador. veronicaolalla@indoamerica.edu.ec, https://orcid.org/0000-0003-3283-5224.
\end{abstract}


another investigation, to propose strategies that direct students to improve their techniques and strategies to improve their learning

Keywords: Self-management of learning, stages of self-management, on-line learning modality

\section{Resumen.}

Introducción. Debido a la pandemia que actualmente impacta a la humanidad por acción del COVID-19, el estilo de vida de las personas ha cambiado drásticamente, uno de los sectores afectados mayormente es el educativo, por esta razón los estudiantes programaron en buscar mejores estrategias de aprendizaje. Objetivo. Este trabajo tuvo como principal propósito realizar un análisis de la autogestión del aprendizaje del estudiante de grado, un caso de estudio en la modalidad a distancia Metodología. Se aplicó una encuesta de 33 preguntas para conocer la autogestión del aprendizaje de los estudiantes, dividida en cuatro secciones: planificación, seguimiento, control y motivación. Resultados. Como principales resultados se obtuvo que los estudiantes de niveles superiores aplican mejores estrategias para la autogestión del aprendizaje que los estudiantes de los niveles medio e inferior. Conclusión. Los estudiantes de niveles inferiores y medio no tienen estrategias que ayuden a mejorar su autoaprendizaje, por lo cual es necesario en otra investigación, plantear estrategias que direccionen a los estudiantes a mejorar sus técnicas y estrategias para mejorar su aprendizaje.

Palabras claves: Autogestión del aprendizaje, etapas de autogestión, modalidad a distancia.

\section{Introducción.}

En la actualidad, el contexto asociado a la pandemia por COVID-19, ha afectado a la población humana y a sus actividades cotidianas incluida la educación, es por ello que las universidades a nivel mundial han requerido de transformaciones en la enseñanza, de lo habitual a una educación virtual, lo que ha influenciado en cambios en las estrategias de aprendizaje y actividades metodológicas (Dwidienawati, 2021; Caballero-Riera, Martínez-Nariño, Guillen-Cerpa, \& Cos-Parlay, 2020).

Las diferentes modalidades de estudio ofertadas en educación superior ocasionan que sea, tanto el estudiante como la institución académica, las que busquen diferentes herramientas para el desarrollo y fortalecimiento del aprendizaje. En este último año, el auto estudio y la auto gestión del aprendizaje juegan un papel fundamental para alcanzar los retos anhelados en cada modalidad de estudio, los mismos que han transformado la educación presencial y semipresencial a una educación en línea, híbrida y a distancia.

La educación a distancia, virtual o híbrida en el ciclo superior, tiene como fin que el estudiante desarrolle a más de su conocimiento, sus capacidades con valores de manera 
más autónoma, insertándose en una educación activa, bidireccional y virtual para que sea capaz de crear y transformar de manera significativa un problema real del contexto (Medina Sánchez, 2021).

Los hábitos de estudio se fomentan desde los primeros años de vida y mucho depende de la capacidad del estudiante para organizar su vida académica, personal y profesional y alcanzar sus objetivos, el estudiante internamente desde su rol, no solo requiere estar sujeto a un nuevo aprendizaje sino a desarrollar una capacidad crítica, constructiva, innovadora y cognitiva, en la que a través de su propia autogestión sea capaz de aprender a conocer, aprender a ser, aprender a convivir, aprender a actuar, aprender a decidir (Estrada, Fuentes, \& Blanco, 2018; Vargas, 2002; Chaves Barboza, 2014).

Desde el punto de vista educativo se puede definir que la autogestión está determinada como un proceso a través del cual no solo se modifican los conocimientos, sino que va más allá de el solo hecho de desarrollar las diferentes habilidades que poseen cada uno de los estudiantes para educarse por sí mismos (Ramos, y otros, 2020), así, este logro y avance que realiza el estudiante al momento de educarse es un efecto del estudio, la experiencia, la instrucción, el razonamiento y la observación que se vive en el aula en el proceso de enseñanza y aprendizaje (Chaves Barboza, 2014; Naranjo, 2009; Barría, Rodríguez, \& Salmerón, 2017; García Gascón, Del Toro Añel, Cisneros Prego, \& Querts, 2017)

El estudiante es el que puede gestionar su propio aprendizaje a través de la capacidad de comprender y aplicar estrategias para su conocimiento. Según Fermín, Fuentes y Soria (2017), el gestionar el aprendizaje es un término que está definido y determinado por el proceso en el cual, la construcción de un aprendizaje formal se basa en lograr metas propuestas por los estudiantes a través de una educación personalizada y dirigida basada en la motivación y conducta propia, la autogestión en el aprendizaje permite al estudiante el desarrollo de las diferentes habilidades que consolidan los aprendizajes dentro o fuera del aula de clases (Fernández Cueli, García, \& González-Castro, 2013; Calcines Castillo, Valdés Utrera, \& Monteagudo de la Guardia, 2017; Stover, Uriel, Freiberg Hoffmann, \& Fernández Liporace, Estrategias de aprendizaje y motivación académica en estudiantes universitarios de Buenos Aires, 2015).

\section{Desarrollo}

Aprender requiere de experiencias vividas y nuevas, en las que se involucra el contexto, la conducta y la motivación, en el que se pueden modificar no solo los conocimientos, sino las habilidades, destrezas, conductas y valores (Ramos-Galarza, y otros, 2020), es así que, al existir factores asociados que impidan gestionar el autoaprendizaje puede repercutir en situaciones de deserción, rezago académico y desmotivación por la construcción de un nuevo aprendizaje (Núñez-Naranjo, 2019; Nuñez-Naranjo, 2020). Gestionar el propio aprendizaje requiere de cuatro etapas, planificación, seguimiento, control y motivación a ello se asocia variables por las que un estudiante toma la decisión de continuar o abandonar su objetivo o meta académica (Núñez Naranjo, Becerra García, \& Olalla Pardo, 2021). 
Planificación. - A la planificación se la conoce como el proceso de establecer metas y elegir medios para alcanzar dichas metas (Barriga, 2009). Jiménez (1982) define a la planificación como el proceso de toma de decisiones para alcanzar un futuro deseado, teniendo en cuenta la situación actual y los factores internos y externos que pueden influir en el logro de los objetivos, por su lado Westreicher (2020) agrega que, la planificación es la estructuración de una serie de acciones que se llevan a cabo para cumplir determinados objetivos, lo que implica, que el sujeto está en la capacidad de tomar sus propias decisiones con el propósito de mejorar la calidad de vida anticipándose a eventos que puedan ser mejorados. El proceso de planificar está determinado en la habilidad que posee cada estudiante para organizar su tiempo y establecer hábitos de estudio que mejoren los procesos educativos, los entornos de aprendizaje influyen en los procesos educativos (Rodríguez, 2018; Westreicher, 2020).

En la etapa de planificación el estudiante debe considerar los medios que va a utilizar para alcanzar sus metas u objetivos, su formación académica inicia en los primeros niveles acompañados de una buena planificación, la distracción por actividades sociales y de ocio son factores contraproducentes para el óptimo desempeño del estudiante (Pérez, Valenzuela Castellanos, Diaz, \& Carlos, 2013).

Control. El control está definido como un proceso que asegura que cada uno de los recursos sean usados de manera efectiva, eficiente y eficaz para el logro de los objetivos que se plantea cada estudiante, para Scanlan (1988) el control tiene como objeto cerciorarse de que las actividades se desarrollen de acuerdo a los planes establecidos, mientras que para Robbins (1996) el control es un proceso en el cual se puede regular el cumplimiento de las actividades planificadas.

La etapa de control permite vincular actividades, para la autogestión del aprendizaje como son: los recursos y estrategias, vinculado con los cambios cualitativos y cuantitativos en la formación de los estudiantes, en el mismo hecho de saber cuándo y cómo aprender además de la importancia de realizar procesos de autoevaluación de resultados para la toma de decisiones (Caballero, 2020).

Seguimiento. - Para Sánchez (2020), el seguimiento corresponde a la necesidad de mejorar el proceso educativo orientado a la evaluación de los diferentes planes y proyectos educativos, además está relacionado con la identificación y el análisis de los distintos subsistemas que conforman la estructura educativa, por tanto, se puede hacer referencia que el proceso de la autogestión del aprendizaje y la calidad de la educación, por medio de factores educativos, promueven la investigación, participación y acción de los diferentes recursos que posee el estudiante (Estévez \& Pérez, 2007).

Para Ostos (2020), las etapas de seguimiento y control permiten al alumno conocer el tiempo dedicado a cada actividad académica, por ejemplo, las horas semanales dedicadas a su carga académica, si presenta problemas para avanzar en el curso, si va entendiendo los contenidos que estudia y si va superando las tareas que se le han propuesto. Raya (2010) afirma que, para tener buenos aprendizajes académicos y resultados óptimos, lo que influye en el estudiante, son los conocimientos previos, las técnicas y estrategias de 
estudio, las aptitudes intelectuales y la motivación social, sin embargo según Herrera (2020), la escasa utilización de técnicas de aprendizaje en estudiantes de educación superior, se debe a la poca responsabilidad y compromiso dentro del ámbito académico, debido a que no aplican estrategias para el aprendizaje.

Motivación. - La motivación se considera, como un proceso que lleva a las personas hacia un fin dentro de un objetivo propuesto, el mismo que implica la existencia de metas (Carreño \& Toscano, 2012), al mismo tiempo está determinado como la energía, dirección, persistencia y finalidad de los comportamientos de las personas (Stover, Uriel, Freiberg Hoffmann, \& Fernández Liporace, 2015). La motivación es considerada como un proceso interactivo y adaptativo con factores cognitivos, afectivos y emocionales, que regulan el organismo y dirigen al logro de sus objetivos establecidos desde el punto de vista educativo (León Urquijo, Risco del Valle, \& Alarcón Salvo, 2014).

García y Ripa (2016), afirman que las habilidades motivacionales son un factor determinante en el aprendizaje y el rendimiento académico, en vista de que aumentan la eficacia de las técnicas empleadas y facilitan la realización de tareas académicas. La actitud de los estudiantes ante diferentes eventos ha sido siempre un aspecto importante en su desarrollo personal y académico, por ello la motivación es muy importante en el momento de aprender dado que está ligado directamente con la disposición del estudiante y el interés en el aprendizaje, mientras más motivado esté, más aprenderá y llegará fácilmente a un aprendizaje significativo (González, 2006).

Boza (2012), considera que las estrategias que se utilicen para la autogestión del aprendizaje, son un factor que influye en la motivación, así Tejedor (2003) resalta que la importancia de la motivación en el momento de aprender está ligado directamente con la disposición del estudiante y el interés en el aprendizaje, ya que sin el trabajo del estudiante no serviría de mucho la actividad del docente, por lo cual se considera que mientras más motivado está el estudiante, más aprenderá. Estos autores resaltan la importancia de las estrategias para la autogestión del aprendizaje en la educación universitaria, puesto que permiten el avance y desarrollo del estudio autónomo a más de potenciar las capacidades individuales de cada estudiante, mejoran el rendimiento académico y enriquecen los procesos de aprendizaje.

El proceso del autoaprendizaje se fundamenta en una filosofía humanista, donde los estudiantes desarrollan la capacidad natural de aprender, para buscar su autorrealización y el interés por lograr un aprendizaje significativo (Torrano, 2007). De la misma manera, en relación a lo mencionado Mondragón (2017) considera que el aprendizaje y el rendimiento académico, se encuentran beneficiados por elementos como la planificación, los hábitos de estudio y factores socio-afectivos.

Otro investigador como Casé (2012), plantea diferencias relevantes entre las técnicas de aprendizaje utilizadas por estudiantes de niveles inferiores, con relación a los niveles superiores, menciona, que no existen estrategias adecuadas para lograr los objetivos planteados, finalmente León (2014), afirma que el poco o nulo uso de estrategias para la autogestión del aprendizaje causan dificultades en la memorización, concentración, 
planificación y análisis de situaciones específicas, por lo que el aprendizaje significativo se ve afectado.

\section{Método}

El estudio se realizó en una universidad particular, en la Carrera de Educación Básica Modalidad a Distancia, la población de estudio correspondió a 199 estudiantes de los niveles inferior, medio y superior, clasificados en 49 hombres y 150 mujeres, con edades que fluctúan entre 17 y 50 años, algunos estudiantes que han retomado sus estudios entre uno y diez años posteriores al haber cursado la secundaria.

Este trabajo tiene un enfoque mixto, es cualitativo porque se describió y analizó la autogestión del aprendizaje de los estudiantes, tomando en cuenta las experiencias y opiniones subjetivas de los mismos, lo que permitió tener un orientación más clara de la investigación y es cuantitativo porque se utilizó herramientas informáticas y matemáticas para obtener resultados, este trabajo está enmarcado en la investigación acción formativa, porque llevó un proceso práctico que inició con el desarrollo y aplicación de una encuesta. El diseño del trabajo es descriptivo explicativo, porque describe los componentes principales de la realidad de los estudiantes entorno a la autogestión del aprendizaje y explica la relación causal e intenta que permitió establecer comparaciones entre las etapas de la autogestión: planificación, seguimiento, control y motivación.

El proceso metodológico de la recopilación de la información sobre la autogestión del aprendizaje en estudiantes se centró en aplicar como técnica una encuesta y el instrumento un cuestionario estructurado en cuatro secciones: planificación, seguimiento, control y motivación, con un total de 33 preguntas divididas de la siguiente manera: 8 de planificación, 7 de seguimiento, 9 de control y 9 de motivación. La aplicación del instrumento se realizó a través de las herramientas digitales web 2.0. La validación del instrumento fue realizada por estudiantes y docentes de la universidad como pares académicos por la experiencia y el conocimiento, demás se basó en la revisión de la literatura publicada por (Núñez Naranjo, Becerra García, \& Olalla Pardo, 2021).

Con base lo expuesto, el procedimiento quedó definido primero por la estructuración de una encuesta para conocer cómo gestionan los estudiantes su aprendizaje, en cuatro etapas: planificación, seguimiento, control y motivación, como segundo punto. Se aplicó la entrevista a estudiantes de los niveles inferior, medio y superior, en tercer lugar, se procedió al análisis de la información, en cuarto lugar, se estableció comparaciones de la información por niveles y para finalizar, se estableció conclusiones, entre estas se propone establecer nuevas estrategias para la autogestión.

\section{Resultados}

Para conocer el nivel de autogestión de aprendizaje de los estudiantes de la modalidad a distancia en las carreras de Educación Básica, se aplicaron encuestas a un grupo 
poblacional conformado por 199 estudiantes, de ellos 62 correspondieron a los primeros niveles, 65 a niveles intermedios y 72 a niveles superiores.

Análisis del Nivel inferior.- Los estudiantes de estos niveles, no presentaron actitudes positivas hacia el autoaprendizaje. En la etapa de planificación el $61 \%$ de los estudiantes presentan dificultades para planificar sus actividades académicas, esto está asociado a los escasos recursos tecnológicos y su contexto. En la etapa de seguimiento un $40 \%$ mencionó no revisar periódicamente sus actividades académicas y los recursos materiales audiovisuales y multimediales subidos a las plataformas virtuales. En la etapa de control el $45 \%$ afirmó no tener un control de sus actividades académicas por presentar, así como no indicaron no solicitar tutorías de refuerzo para retroalimentación y mejora del rendimiento académico o por inasistencia. En la etapa de la motivación, un $78 \%$ se motiva por el uso de las diferentes herramientas tecnológicas, material audiovisual y multimedial, así como por la búsqueda de información en otras fuentes bibliográficas, mientras que el $60 \%$ desconoce los nuevos entornos tecnológicos y uso de herramientas tecno pedagógicas que facilitan el autoaprendizaje.

Análisis del nivel medio. -En este nivel los estudiantes presentaron una actitud no tan favorable hacia el autoaprendizaje, en la etapa de planificación se tuvo como principales resultados que el 58\% no planifica sus actividades académicas, esto puede estar influenciado debido a que no siempre cuenta con acceso al internet lo que dificulta conocer fechas de entregas de tareas y planificarlas para la entrega y el $28 \%$ indicó que nunca realizan un listado de actividades pendientes. En la etapa de seguimiento, un 33\% presenta problemas hacer seguimiento a sus actividades académicas pendientes, además indicaron que no repasan el material guía ni revisan la retroalimentación, así como no cumple con la entrega de tareas dentro de los tiempos establecidos posponiendo aquellas que les dificulta. En la etapa de control, el 35\% no lleva un registro de control de sus actividades académicas, presenta dificultades al momento de liderar trabajos en equipo, les cuesta organizar sus actividades comunes y separar las académicas. En la etapa de motivación, un $58 \%$ está motivado por las herramientas tecnopedagógicas recursos multimediales y audiovisuales, mientras que el $42 \%$ no busca información adicional en el internet o en bibliografía o web grafía, y mencionan que subestiman el tiempo, así como deja para el final las tareas que le resultan más complicadas.

Análisis el nivel superior. - Los estudiantes de este grupo, presentaron una condición positiva frente al autoaprendizaje, el $73 \%$ indicó llevar una agenda de planificación de actividades académicas, y apenas el $27 \%$ de los estudiantes indicaron no contar con recursos tecnológicos permanentes que les permita llevar una planificación conforme sus actividades. En la etapa del seguimiento un 51\% no hace seguimiento a la planificación de sus actividades académicas, no revisa el aula virtual y la retroalimentación constantemente. En la etapa de control el 34\%, menciono tener control de la planificación de sus actividades académicas, llevando un registro de sus actividades cumplidas y pendientes. En la etapa de la motivación, el 64\% mencionó estar motivado por los diferentes recursos didácticos e interactivos subidos en el aula que les permite fortalecer su auto aprendizaje, así como por el propósito de alcanzar de su meta profesional, y el 
$88 \%$ indicó que busca información adicional en el internet o en bibliografía o web grafía para mejorar su aprendizaje

Comparación de datos obtenidos en los diferentes niveles.- Según el análisis de los resultados por niveles, en la etapa de planificación los estudiantes de los niveles inferiores y medios presentan mayores dificultades al momento de planificar, debido a no llevar un plan de trabajo académico y al poco conocimiento en el uso de herramientas digitales, mientras que los estudiantes de niveles superiores, presentan mayor responsabilidad con la planificación de sus actividades académicas.

En la etapa de seguimiento, los estudiantes de los niveles inferiores y medios presentan menores estrategias para realizar seguimiento a sus actividades académicas, esta particularidad puede estar relacionada al reciente ingreso a la vida universitaria, mientras que los estudiantes de niveles superiores realizan mayor seguimiento a sus actividades académicas influenciado por la decisión de obtener el título de tercer nivel y el mayor conocimiento de la modalidad a distancia.

En la etapa de control los estudiantes de los niveles inferiores y medios presentan menores habilidades de control de sus actividades académicas, que los estudiantes de los niveles superiores, situación que está asociada a llevar un plan de trabajo semestral según su planificación, además de que la constancia permite fortalecer su rutina diaria

En la etapa de la motivación los estudiantes de los niveles inferiores se encuentran más motivados que los estudiantes de los niveles inferiores, esto puede ser ocasionado por los diferentes recursos multimediales y audiovisuales de interés del dicente de reciente ingreso, al uso mismo de nuevas plataformas virtuales.

Se aprecia que las etapas de autogestión del estudiante universitario como la planificación, seguimiento y control van potenciándose mientras el estudiante avanza al siguiente nivel, mientras que la etapa de motivación disminuye.

\section{Conclusiones}

- A partir de esta investigación se logró conocer los diferentes elementos que involucran el auto aprendizaje en un estudiante universitario en sus cuatro etapas que inciden en su actividad y rol estudiantil autónomo, considerándose de relevancia en el quehacer educativo ya que el aprender de una manera organizada, consciente y auto-gestionada permite un mejor desenvolvimiento académico.

- Las investigaciones que relacionan los factores de mayor incidencia que afectan la autogestión del aprendizaje, son la inteligencia, motivación y estrategias individuales, las cuales, a pesar de ser estudiadas en otros trabajos, no son posibles de determinar con exactitud, su relación con el objeto de estudio de esta investigación, al ser variables de constructo multidimensional y al carecer de instrumentos de medida exactos.

- Los estudiantes de los niveles superiores presentan mejores estrategias para la autogestión del aprendizaje en las etapas de la planificación, seguimiento y 
control, llevan una agenda, utilizan de mejor manera las herramientas virtuales, organizan y presentan sus tareas a tiempo además llevan un control del cumplimiento de las tareas, buscan información relevante en fuentes de bibliográficas lo que facilita el mejor el cumplimento sus actividades académicas, sin embargo, etapa de motivación a relación de los otros niveles es baja.

- Los estudiantes de los niveles inferiores y medios presentan mayores dificultades para autogestionar su aprendizaje a ello la importancia de implementar estrategias que les permita mejorar su aprendizaje autónomo

\section{Referencias Bibliográficas}

Barría, C., Rodríguez, S., \& Salmerón, P. (2017). Autorregulación del aprendizaje en centros educativos de Granada donde se utilizan las Tecnologías de la Información y la Comunicación. REIDOCREA, 140-155. doi:ISSN: 2254-5883

Barriga. (2009). La planificación. Obtenido de http://www. geocities. com/WallStreet/District/7921/Planification. html. Bishwapriya Sanya. Planning as anticipation of resistance en: http: www. seweb. uci. edu/ppd/ufiles/Sanyal\% 20Resistanc

Boza, Á. \&. (2012). Motivos, actitudes y estrategias de aprendizaje: aprendizaje motivado en estudiantes universitarios. evista de currículum y formación del profesorado, 16(1), 126-142.

Caballero, L. O. (2020). La autogestión del aprendizaje, su desarrollo desde la orientación, control y evaluación de trabajo independiente. MAESTRO $Y$ SOCIEDAD . doi:1815-4867, 17(1)

Caballero-Riera, O., Martínez-Nariño, Z., Guillen-Cerpa, S., \& Cos-Parlay, Y. (2020). La autogestión del aprendizaje, su desarrollo desde la orientación, control y evaluación de trabajo independiente. Maestro y Sociedad: Revista electrónica para maestros y profesores, 17(1), 39-51. Obtenido de https://maestroysociedad.uo.edu.cu/index.php/MyS/article/view/5145/4607

Calcines Castillo, M., Valdés Utrera, J., \& Monteagudo de la Guardia, R. (2017). La autogestión del conocimiento: ¿una meta inalcanzable? EDUMECENTRO, 9(2), 225-229. doi:ISSN 2077-2874

Carreño, Á., \& Toscano, M. (2012). Motivos, actitudes y estrategias de aprendizaje: aprendizaje motivado en alumnos universitarios. Revista de currículum y formación de profesorado. doi:1989-639X

Casé, L. N. (2012). strategias de aprendizaje y rendimiento académico según el género en estudiantes universitarios. Revista de psicología, . 
Chaves Barboza, E. (2014). Autogestión del aprendizaje en la investigación educativa sobre Entornos Personales de aprendizaje (PLE): Una revisión de literatura. EDEMIC, 3(2), 114-134. doi:https://doi.org/10.21071/edmetic.v3i2.2892

Dwidienawati, D. (2021). Revisit the implementation of E-Learning: Lesson Learned from COVID-19 pandemic. Revista Virtual Universidad Católica del Norte(63), $1-4$.

Obtenido

de https://www.redalyc.org/jatsRepo/1942/194266612001/194266612001.pdf

Estévez, J., \& Pérez, M. (2007). Sistema de indicadores para el diagnostico y seguimiento de la educacion superior en México . Diseno Gráfico y Armado Editorial . doi:970-704-103-X

Estrada, O., Fuentes, D., \& Blanco, S. (2018). Estrategia para la formación profesoral en el autoaprendizaje estudiantil. Opuntia Brava, 10(4), 7-8. doi:ISSN: 2222-081x

Fermín, T., Fuentes, J. L., \& Soria, M. (2017). Aprendizaje autorregulado: estado de la cuestión y retos psicopedagógicos. Perfiles educativos. doi:0185-2698

Fernández Cueli, M., García, T., \& González-Castro, P. (2013). Autorregulación y rendimiento académico en Matemáticas. Dianet, 41(1), 39-48. doi: ISSN 02102773

García Gascón, A., Del Toro Añel, A. Y., Cisneros Prego, E., \& Querts. (2017). García Gascón, Angel; Del Toro Añel, Annia Yolanda; Cisneros Prego, Elio; Querts. MEDISAN. doi:1029-3019

García-Ripa, M. I.-G. (2016). Estrategias de Aprendizaje y Autorregulación Motivacional. Identificación de Perfiles para la Orientación de Estudiantes Universitarios de Nuevo Ingreso. Revista Iberoamericana de Diagnóstico y Evaluación.

González, D. \&. (2006). La importancia de promover en el aula estrategias de aprendizaje para elevar el nivel académico en los estudiantes de Psicología. Revista Iberoamericana de educación, 40(1), 1-17. doi: https://doi.org/10.35362/rie4012532.

Herrera, L. \&. (2020). Estrategias de aprendizaje en estudiantes universitarios. (EDUC, Ed.) Un aporte a la construcción del Espacio Europeo de Educación Superior, 12(13), 75-98.

Jiménez. (1982). Introducción al Estudio de la Teoría Administrativa. Mexico: Editorial FCE.

León Urquijo, A. P., Risco del Valle, E., \& Alarcón Salvo, C. (2014). Estrategias de aprendizaje en educación superior en un modelo curricular por competencias. REVISTA DE LA EDUCACION SUPERIOR . doi:0185-2760 
León, A. R. (2014). Estrategias de aprendizaje en educación superior en un modelo curricular por competencia. Revista de educación superior, 43(4), 123 - 144.

Medina Sánchez, Y. (2021). La gestión del aprendizaje como herramienta formativa en el sistema educacional cubano actual. Maestro y Sociedad, 18(2), 802-814. Obtenido de https://maestroysociedad.uo.edu.cu/index.php/MyS/article/view/5376/5011

Mondragón, C. C. (2017). Hábitos de estudio y rendimiento académico. Caso estudiantes de la licenciatura en Administración de la Unidad Académica Profesional Tejupilco. Revista Iberoamericana para la investigación y el desarrollo educativo, 8(15), 5,6,7,. doi:10.23913/ride.v8i15.315

Naranjo, M. (2009). Motivación perpestivas teóricas y algunas consideraciones de su importancia en el ámbito educativo. Eduación, 23.

Núñez Naranjo, A. F., Becerra García, E. B., \& Olalla Pardo, V. E. (2021). Autogestión del aprendizaje: Revisión de la literatura. Explorador DIgital, 5(2), 6-22. doi:https://doi.org/10.33262/exploradordigital.v5i2.1649

Núñez-Naranjo, A. (2019). Deserción y estrategias de retención: un análisis desde la universidad particular. 593 Digital Publisher CEIT, 5((5-2)), 78-87. doi:https://doi.org/10.33386/593dp.2020.5-2.306

Nuñez-Naranjo, A. (2020). Deserción y estrategias de retención: un análisis desde la universidad particular. 593 Digital Publisher CEIT, 5(5-2), 79-87. doi: https://doi.org/10.33386/593dp.2020.5-2.306

Ostos, O. (2020). Gestión del conocimiento, un reto en la educación superior. CRAIUSTA. doi:https://doi.org/10.15332/dt.inv.2020.00870

Pérez, M. V., Valenzuela Castellanos, M., Diaz, A. G., \& Carlos, N. J. (2013). Dificultades de aprendizaje en estudiantes universitarios de primer año. doi:07180462

Ramos, C., Rubio, D., Ortiz, D., Acosta, P., Hinojosa, F., Cadena, D., \& López, E. (2020). Autogestión del aprendizaje del universitario: un aporte en su construcción teórica. Revista ESPACIOS. doi:ISSN 07981015

Ramos-Galarza, C., Rubio, D., Ortiz, D., Acosta, P., Hinojosa, F., Cadena, D., \& López, E. (2020). Autogestión del aprendizaje del universitario: un aporte en su construcción teórica. Espacios, 41(18), 1-14. Obtenido de https://www.revistaespacios.com/a20v41n18/20411816.html

Raya Ramos, E. (2010). Factores que intervienen en el aprendizaje. Revista digital para profesionales de la enseñanza, 6, 1-6. Obtenido de https://www.feandalucia.ccoo.es/docu/p5sd7060.pdf. 
Robbins. (1996). Administración. México, D.F.: Pearson Educación.

Rodríguez, S. P. (2018). Bienestar emocional de los estudiantes universitarios: el papel de la orientación a metas y las percepciones de control. Universidad de Granda . doi:http://dx.doi.org/10.30827/publicaciones.v48i1.7324

Sánchez, M. (2020). Seguimiento y evaluación de planes y proyectos educativos: un reto y una oportunidad. Omnia. doi:1315-8856

Scanlan, B. (1988). El Proceso Administrativo. Obtenido de http://jotvirtual.ucoz.es/ADMON/EL_PROCESO_ADMINISTRATIVO.pdf

Stover, J. B., Uriel, F., Freiberg Hoffmann, A., \& Fernández Liporace, M. (2015). Estrategias de aprendizaje y motivación académica en estudiantes universitarios de Buenos Aires. Dialnet. doi:2451-6600

Stover, J. B., Uriel, F., Freiberg Hoffmann, A., \& Fernández Liporace, M. (2015). Estrategias de aprendizaje y motivación académica en estudiantes universitarios de Buenos Aires. DIALNET Obtenido de https://dialnet.unirioja.es/servlet/articulo?codigo=5645334

Tejedor Tejedor, F. J. (2003). Poder explicativo de algunos determinantes del rendimiento en los estudios universitarios. Revista Española de Pedagogía, 224, 5-32. Obtenido de https://dialnet.unirioja.es/servlet/articulo?codigo $=718488$

Torrano, F. F. (2007). Aprendizaje autorregulado: estado de la cuestión y retos psicopedagógicos. Perfiles educativos, 39(156), 160 - 173.

Vargas, M. E. (2002). El aprendizaje de la ciencia y de la información científica en la educación superior. Revista cientifica española, 3-15. Obtenido de https://revistas.um.es/analesdoc/article/view/2151

Westreicher, G. (2020). Economipedia.com. Obtenido de Economipedia.com.

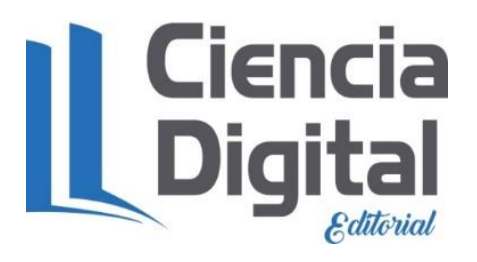




\section{PARA CITAR EL ARTÍCULO INDEXADO.}

Becerra García, E. B., Núñez Naranjo, A. F., \& Olalla Pardo, V. E. (2021). Análisis de la autogestión del aprendizaje del estudiante de grado: un caso de estudio en la modalidad a distancia ConcienciaDigital, https://doi.org/10.33262/concienciadigital.v4i3.1.1811

\section{LCiencia}

El artículo que se publica es de exclusiva responsabilidad de los autores y no necesariamente reflejan el pensamiento de la Revista Conciencia Digital.

El artículo queda en propiedad de la revista y, por tanto, su publicación parcial y/o total en otro medio tiene que ser autorizado por el director de la Revista Conciencia Digital.

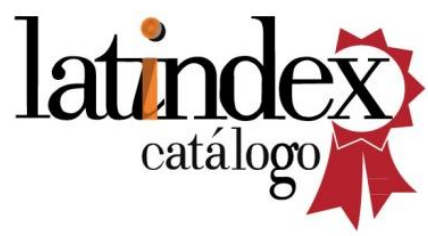

